\section{Spectroscopic Indications of an Internal Spiral Arm in the Galaxy}

Sprral structure within our galaxy has been detected by three methods: (a) space-plotting of stellar associations ${ }^{1}$, (b) multiple structure in the $H 21 \mathrm{~cm}$. radiation ${ }^{2}$, (c) multiple structure in the interstellar lines of $\mathrm{Ca}$ II and Na I in distant stars ${ }^{3}$. The radio method $(b)$ has the advantage over the two optical methods in that it will penetrate to the limits of the galaxy. On the other hand, the interpretation of the radio profiles for directions within $90^{\circ}$ of the galactic centre is greatly complicated by the superposition of radiation from regions at very different distances which have the same angular velocity. By contrast, method (c) can reveal unambiguously the presence of one, two or more spiral arms within a light-path limited by a distant star. The limited light-paths used in method (c) are of advantage in the internal regions of the galaxy.

In the course of work at the Radcliffe Observatory on velocities of southern $B$ stars, certain stars with distances probably greater than 3 k.parsecs wero selected for study at a dispersion of $19 \mathrm{~A} . / \mathrm{mm}$. at $3933 \mathrm{~A}$., with the view of possible resolution of the interstellar $K$ line into components due to spiral arms. Among the five stars listed below, three have been found to have two well-separated $K$ lines, whereas in the other two $K$ is seen to be resolved with a separation which cannot be reliably measured but which is of order $25 \mathrm{~km}$. $/ \mathrm{s}$.

\begin{tabular}{|c|c|c|c|c|c|c|}
\hline \multirow[b]{2}{*}{$H D$} & \multirow[b]{2}{*}{$m_{v}$} & \multicolumn{2}{|c|}{ Stars with double } & \multicolumn{2}{|c|}{$K$ line } & \\
\hline & & & & Star & sities (l & \\
\hline $\begin{array}{l}142,468 \\
42,565 \\
47,5631 \\
47,35\end{array}$ & $\begin{array}{l}8.1 \\
9.0 \\
8.9\end{array}$ & $\begin{array}{l}295 \cdot 6 \\
295 \cdot 7 \\
299 \cdot 8\end{array}$ & $\begin{array}{l}-1.8 \\
=1.8 \\
-3.0\end{array}$ & $\begin{array}{l}-40 \\
-25\end{array}$ & $\begin{array}{l}=14 \\
-12\end{array}$ & $\begin{array}{l}-84 \\
-75\end{array}$ \\
\hline $\begin{array}{l}148,546 \\
173,987\end{array}$ & $\begin{array}{l}7 \cdot 8 \\
9.0\end{array}$ & $\begin{array}{l}311 \cdot 1 \\
354 \cdot 6\end{array}$ & $\begin{array}{l}+5.9 \\
-3.7\end{array}$ & $+54:$ & $+1:$ & 43 \\
\hline
\end{tabular}

Four stars in the longitude range $273^{\circ}-289^{\circ}$, namely, $H D 114,340,122,324,125,241,136,239$, have also been examined with the same dispersion but failed to show doubling of the interstellar lines, despite the fact that these stars are probably as distant as those with doublo lines and lie in longitudes more favourable for resolving components due to differential galactic rotation.

Of the five tabulated stars with double $K$, four lie south of the galactic centre. In each case the low-velocity component must correspond largely to the Sagittarius arm. The high-velocity component prosumably corresponds to an interior arm which might be called the Scutum-Norma arm from the constellations in which it is probably seen tangentially. The radio work has already indicated that this arm is seen tangentially in longitude $3^{\circ}$ at a distance of about $5 \mathrm{k}$.parsecs from the centre 4 . No details concerning radio observations at longitude $295^{\circ}$ seem to be available at present ${ }^{5}$.

The negative evidence from the four stars with $l=273^{\circ}-289^{\circ}$ (some of which have been suggested by E. Smith ${ }^{6}$ as belonging to a distant arm) might be taken as supporting the view that the ScutumNorma arm does not extend beyond $290^{\circ}$. However, these stars might lie on the near side of the arm behind an undetectably small amount of the gas associated with tho arm.

The observed $K$ velocities in the three stars $H D 142,468,142,565$ and 173,987 can be used to derive $\Delta \omega(R)$ on the assumption of circular motions. The calculations are rather sensitive to the assumed longitude of the galactic centre and to the corrections for solar motion. But if the standard values are assumed, we find a value of $3.5 \mathrm{~km} . / \mathrm{s} . / \mathrm{k}$.parsec for the low-velocity components (consistent with the predictions of the radio model for the combined effects of the Sagittarius arm and of gas still nearer the Sun) and for the high-velocity components 16 $3 \mathrm{~km} . / \mathrm{s} . / \mathrm{k}$. parsec in agreement with the model for the more distant arm suggested by the radio observations at $l=3^{\circ}$. It is difficult to avoid the conclusion from these observations that in the Scutum-Norma arm the value of $\Delta \omega(R)$ is greater at $l=295^{\circ}$ than at $l=355^{\circ}$. Interpreted literally, this would imply that the Norma branch is nearer than the Scutum branch to the centre of the galiaxy, that is, that this arm is opening out in the direction of rotation. It would be weighting the meagre data too heavily to deduce that this is a property of the arm as a whole. A more plausible suggestion is that in front of the two stars $H D 142,468,142,565$ we are observing a peculiar stream motion of the Ca II gas.

\section{Radeliffe Observatory,}

A. D. Thackeray

Protoria. Sept. 17.

1 Morgan, W. W., et al., Astron. J., 57, 3 (1952).

${ }^{2}$ van de Hulst, H. C., Muller. C. A., and Oort, J. H., Bull. Astro. Inst. Netherlands, No. 452 (1954).

${ }^{3}$ Munch, G., Pub. Astro. Soc. Pacific, 65, 179 (1953).

' Kwee, K. K., Muller, C. A., and Westerhout, G., Bull. Astro. Inst. Netherlands, No. 45.9 (1954).

- Christiansen, W. N., and Hindiman, J. V., Aust. J. Sci. Res., A, 5, 437 (1952). Kerr, F. J., Hindman, J."v., and Carpenter, M. S., Astron. J., 61, 7 (1956).

- Smith, E., Harvard dissertation (1955).

\section{Radioactive Sulphur produced by Cosmic Rays in Rain Water}

IsOTOPES of carbon, hydrogen and beryllium arising from interactions of primary and secondary cosmic-ray particles with the oxygen and nitrogen nuclei of the atmosphere have been recorded'. 'I'wo isotopes of phosphorus due to spallation of atmospheric argon have also been found ${ }^{2}$. We can now add to the list sulphur-35.

Sulphur-35 is a beta-emitter $\left(E_{\max .}=167 \mathrm{keV}.\right)$ with a half-life of 87 days. Because of the low decayenergy, self-absorption of electrons is serious and it is necessary for detection to have samples of high specific activity. As is well $\mathrm{known}^{3}$, rain water often contains appreciable amounts of stable sulphur partly due to ocean spray, and partly to industrial contamination. Samples collected in Bombay have, on the average, large amounts of stable sulphur (about $0.7 \mathrm{mgm}$. per litre). In order to reduce such contamination and thereby obtain samples with higher specific activity, we have used rain water from Banaras and from Kodaikanal. In samples from Banaras, which is far distant from the sea, the sulphur contents were low, although still about $0.27 \mathrm{mgm}$ per litre, and detection was comparatively easy. Further improvement was achieved by obtaining a sample from Kodaikanal, which is well inland, $7,500 \mathrm{ft}$. above sea-level and with much less industrial pollution. This sample contained $0.08 \mathrm{mgm}$. of sulphur per litre. The results for all the samples are given in Table 1. Since our counter is not yet calibrated for soft beta-radiation, the absolute number of atoms may be in error by as much as a factor of two. The counts per hour have been converted into disintegrations/min. and atoms per litre, by taking account of counter geometry, window absorption, selfabsorption, estimated chemical efficiency and decay. 